\title{
Boosting Energy Storage Performance of Low- Temperature Sputtered CaBi2Nb209 Thin Film Capacitors Via Rapid Thermal Annealing
}

Jing Yan

shan dong da xue: Shandong University

Yanling Wang

Amperex Technology Limited

Chun-Ming Wang

Shandong University

Jun Ouyang ( $\nabla$ ouyangjun@qlu.edu.cn )

Qilu University of Technology (Shandong Academy of Sciences) https://orcid.org/0000-0003-24462958

\section{Research Article}

Keywords: Bismuth layer-structured ferroelectrics (BLSFs), Calcium bismuth niobate (CaBi2Nb209), Nanograin films, Rapid Thermal Annealing (RTA), Energy storage, Fatigue-resistance

Posted Date: October 8th, 2020

DOl: https://doi.org/10.21203/rs.3.rs-84796/v1

License: (c) (1) This work is licensed under a Creative Commons Attribution 4.0 International License. Read Full License 


\section{Abstract}

CaBi 2 Nb 209 thin film capacitors were fabricated on SrRuO 3 -buffered Pt(111)/Ti/Si(100) substrates by adopting a two-step fabrication process. This process combines a low-temperature sputtering deposition with a rapid thermal annealing (RTA) to inhibit the grain growth, for the purposes of delaying the polarization saturation and reducing the ferroelectric hysteresis. By using this method, $\mathrm{CaBi} 2 \mathrm{Nb} 2 \mathrm{O}$ 9 thin films with uniformly distributed nanograins were obtained, which display a large recyclable energy density $\mathrm{W}$ rec $\sim 69 \mathrm{~J} / \mathrm{cm} 3$ and a high energy efficiency $\eta \sim 82.4 \%$. A superior fatigue-resistance (negligible energy performance degradation after 109 charge-discharge cycles) and a good thermal stability (from $-170^{\circ} \mathrm{C}$ to $150^{\circ} \mathrm{C}$ ) have also been achieved. This two-step method can be used to prepare other bismuth layer-structured ferroelectric film capacitors with enhanced energy storage performances.

\section{Full Text}

This preprint is available for download as a PDF.

\section{Figures}
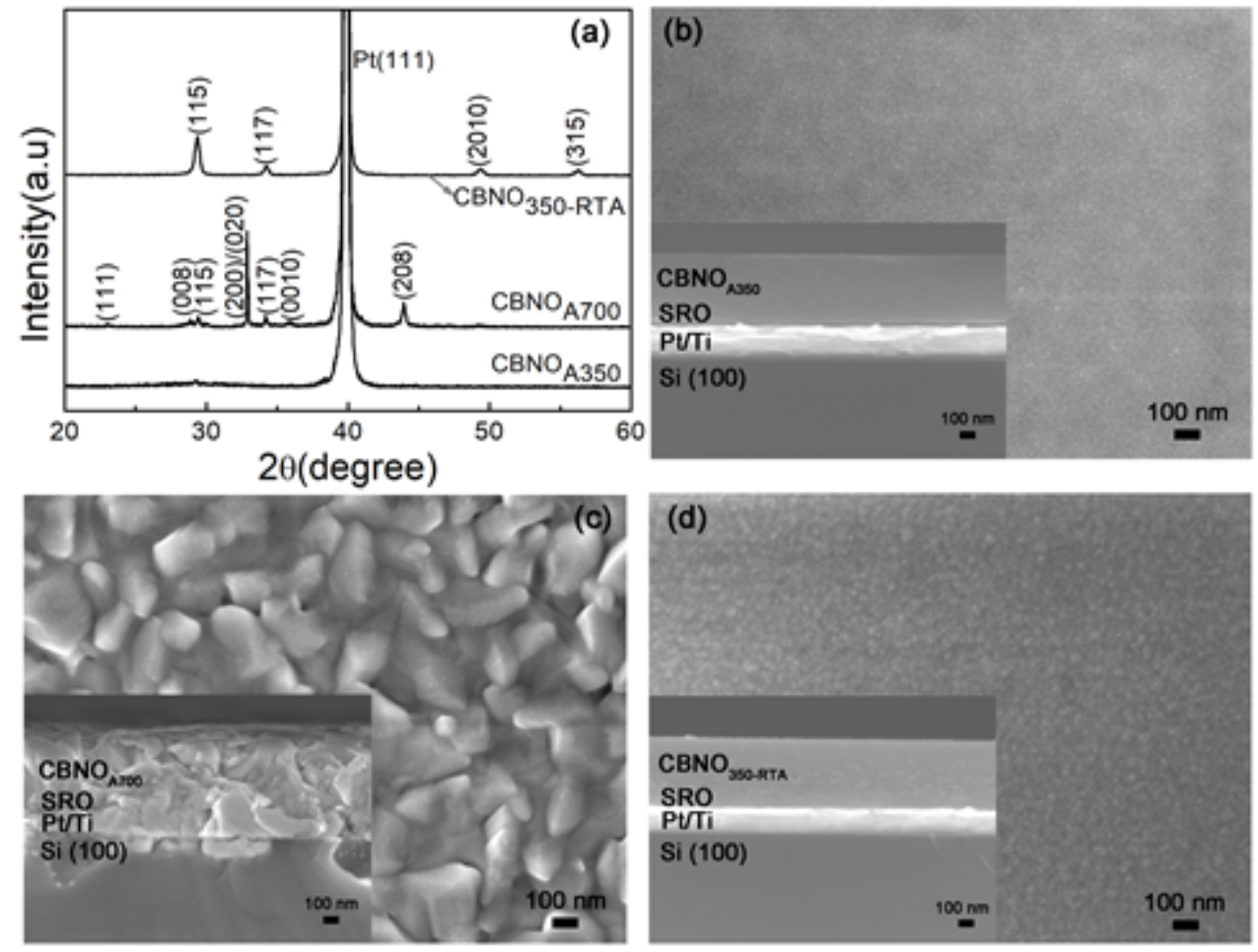

\section{Figure 1}

(a) XRD 20-scan patterns for CBNOA350, CBNOA700, and CBNO350-RTA thin films. (b) (c) (d) The surface and cross-sectional SEM images of the (b) CBNOA350, (c) CBNOA700 and (d) CBNO350-RTA films. 

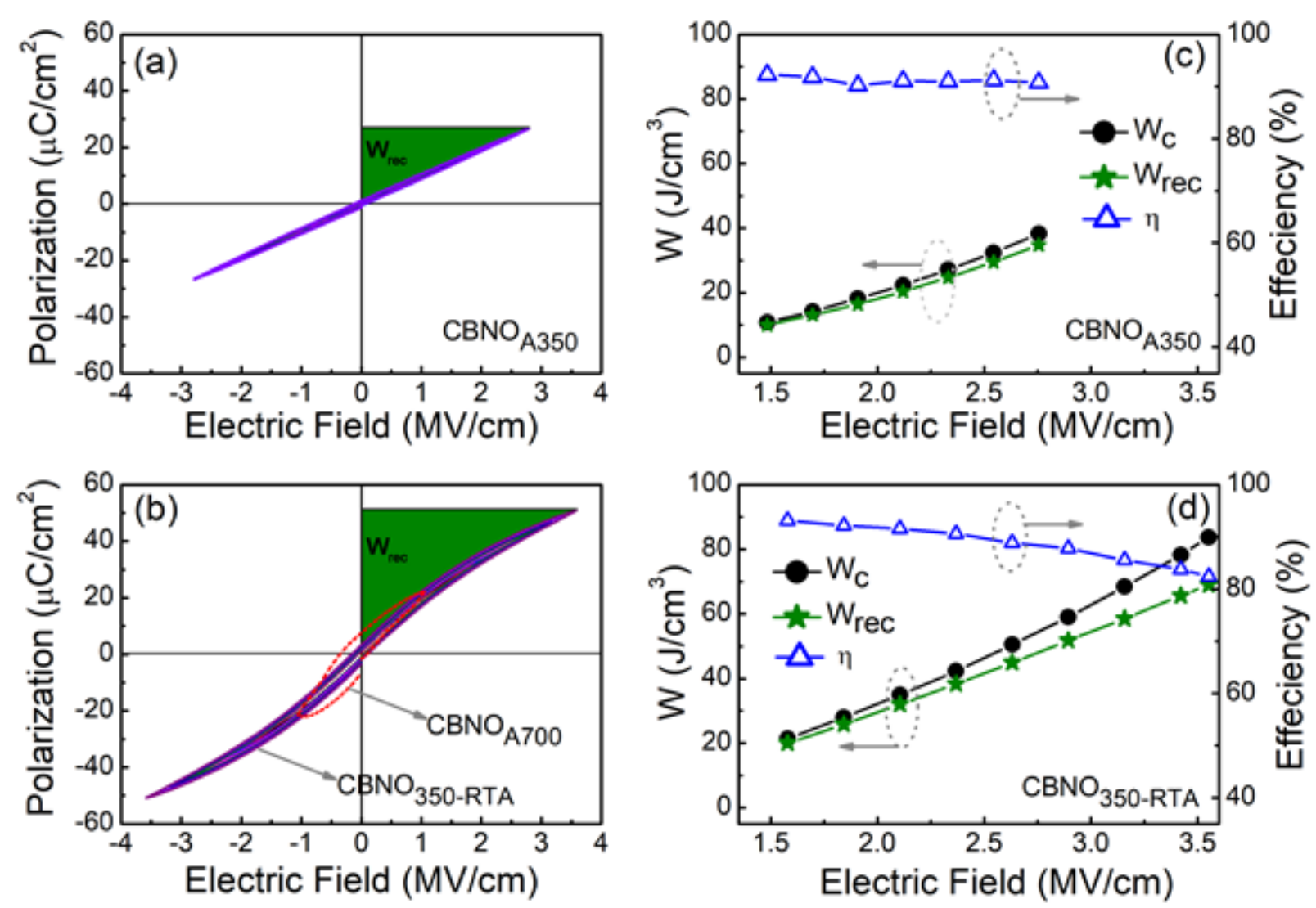

\section{Figure 2}

Room-temperature polarization-electric field (P-E) curves for the (a) CBNOA350 and (b) CBNO350-RTA thin films, and (c) (d) their corresponding energy storage performances. The red dashed-line curve in (b) is a representative P-E loop of the CBNOA700 film at its maximum applicable electric field $(\sim 1 \mathrm{MV} / \mathrm{cm})$. 

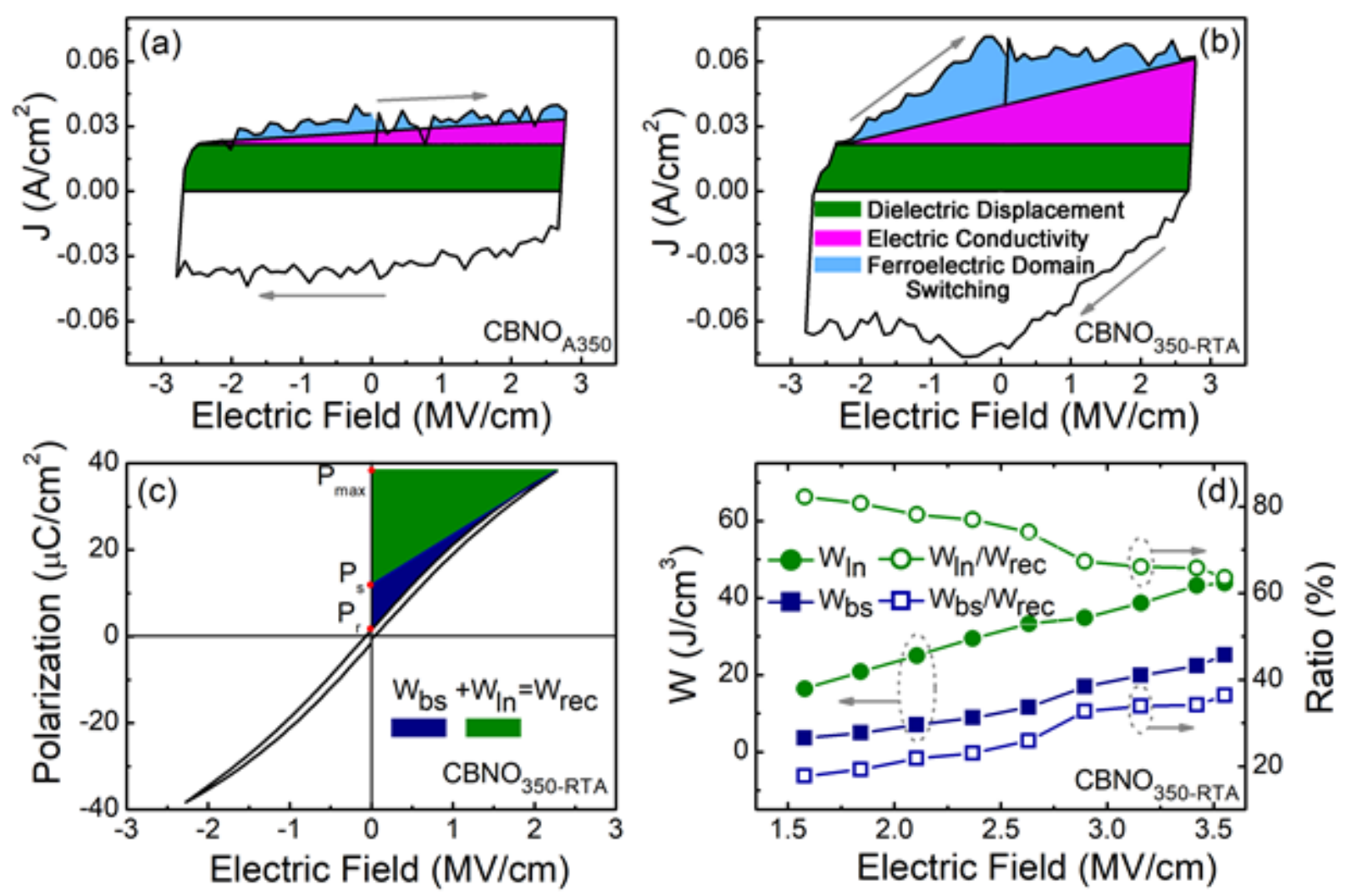

Figure 3

Room temperature (RT) switching current-electric field curves for (a) CBNOA350 and (b) CBNO350-RTA films under an applied electric field of $\sim 2.76 \mathrm{MV} / \mathrm{cm}$. (c) The schematic illustration of the linear dielectric (WIn) and domain backswitching (Wbs) contributions to the recyclable energy density Wrec of the CBNO350-RTA thin film. (d) The contributions from Wln and Wbs to Wrec as functions of the applied electric field (at RT). 

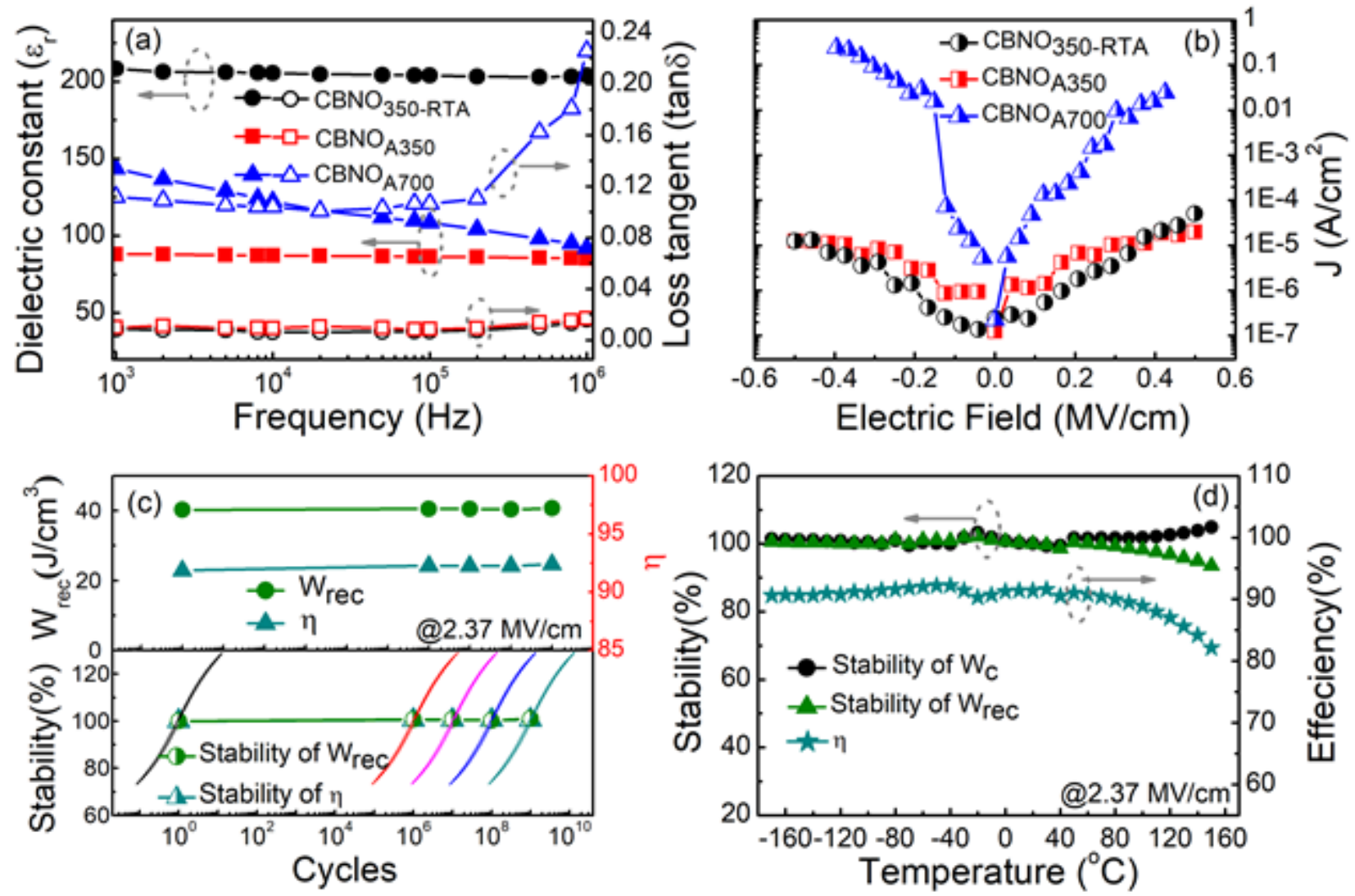

Figure 4

(a) Frequency-dependent dielectric constant ( $\varepsilon r$ ) and loss tangent (tan $\delta$ ) and (b) leakage current density of the CBNOA350, CBNO350-RTA and CBNOA700 films (at RT). (c) Recyclable energy storage density and energy efficiency as functions of charge-discharge cycles (at RT) for the CBNO350-RTA film, and the corresponding P-E hysteresis loops and performance stabilities (Wrec/Wrec-1 stcircle, $\eta / \eta 1$ stcircle ) at different stages of the cycling test. (d) Thermal stabilities (W/WRT, unRT) of Wc, Wrec and uffor the CBN0350-RTA film. 